\title{
Deep learning feature selection to unhide demographic recommender systems factors
}

\author{
J. Bobadilla \\ Á. González-Prieto \\ F. Ortega $\quad \cdot$ R. Lara-Cabrera
}

\begin{abstract}
Extracting demographic features from hidden factors is an innovative concept that provides multiple and relevant applications. The matrix factorization model generates factors which do not incorporate semantic knowledge. Extracting the existing nonlinear relations between hidden factors and demographic information is a challenging task that can not be adequately addressed by means of statistical methods or using simple machine learning algorithms. This paper provides a deep learning-based method: DeepUnHide, able to extract demographic information from the users and items factors in collaborative filtering recommender systems. The core of the proposed method is the gradient-based localization used in the image processing literature to highlight the representative areas of each classification class. Validation experiments make use of two public datasets and current baselines. The results show the superiority of DeepUnHide to make feature selection and demographic classification, compared to the state-of-art of feature selection methods. Relevant and direct applications include recommendations explanation, fairness in collaborative filtering and recommendation to groups of users.
\end{abstract}

Keywords Feature selection · Collaborative filtering $\cdot$ Demographic information · Matrix factorization · Gradient-based localization $\cdot$ Deep learning

\section{Introduction}

Recommender system (RS) [37, 50] are playing an important role in our society: they provide useful information to the users by recommending highly demanded products and services. Remarkable examples of RS are: Amazon, Netflix, TripAdvisor and Spotify. RS are implemented by means of several filtering strategies, mainly the collaborative [37, 50], content [59], demographic [1], context [53] and social [47] ones. Most of the commercial
RS are based on hybrid models that combine collaborative filtering (CF) with some other filtering approaches. In the early ages of RS research, CF was implemented using the K-nearest neighbours $(\mathrm{KNN})$ algorithm [10]: it is easy to understand, to implement and to analyse, since it can be considered as a white-box method. This approach has also been updated and improved in the recent years with promising approaches like hybrid methods [3] or adding information theoretic quality measures [27]. Nevertheless, the KNN main drawbacks are its lack of scalability and its poor accuracy.

Due to the exposed KNN drawbacks, this memory-based algorithm has been replaced by model-based ones, mainly the probabilistic matrix factorization (PMF) [38] and its variations and improvements, such as the nonnegative matrix factorization (NMF) [32], Bayesian NMF (BNMF) [22] of two-level MF (TLMF) [34]. Currently, research is also focusing on deep learning (DL) $[9,25,39]$ based approaches. Model-based approaches are scalable and accurate, but they act as a black box, making it difficult to address some RS goals such as recommendations 
explanation or improvements in the beyond accuracy goals such as fairness, diversity, reliability or serendipity. The explained research evolution on RS is relevant to this paper, since it makes use of an architecture that combines the matrix factorization $(\mathrm{MF})$ and $\mathrm{DL}$ approaches, trying to unhide the MF black box model.

This paper shows a novel research objective compared with the related published works of the authors in the area. In $[8,9]$, the idea of applying deep learning techniques to improve CF-based RS is exploited but right in the core of the CF methods to accurately recommend items to users without the use of MF hidden factors. Other works by the authors are also oriented to give new perspectives to the $\mathrm{CF}$ method, like in $[22,31,42,52]$ where new methods of statistical nature are provided for computing and processing the MF. Finally, the problem of extracting new information from $\mathrm{CF}$ data has also been addressed by the authors in [23], but with a different approach based on the explainability of classification trees. To the best of our knowledge, this is the first attempt to provide semantic interpretation to $\mathrm{CF}$ hidden factors by means of DL techniques.

CF RS datasets are really sparse [11], and MF models make a reduction of dimensionality to obtain compressed and dense versions of them. In the MF models, each user is represented by a reduced number of $K$ factors (real numbers) that encode the user's essence. Each dataset item is represented in the same way. Since the MF dimensionality reduction process makes use of the same vector space, both items and users share the same semantic coded into their factors. Although only ratings are used in the basic MF algorithm, they contain implicit information related with demographic features of the RS users and items; e.g. young female people is usually interested in different types of clothes (items) than old male do. In this way, statistical distributions of ratings are not random at all: RS distributions of ratings implicitly encode semantic information such as the demographic one (gender, age, zip code, etc.). In this sense, MF transfers the ratings semantic information to the obtained hidden factors.

Figure 1 shows the MF basic operational; on its top, the compression is represented, where a sparse matrix of ratings is converted in two dense matrices of factors: the users' and the items' ones. To predict the rating of a user to an item, the dot product is used; recommendations to each user are just those items with the best predictions. The bottom of Fig. 1 shows the essence of the dot product: predictions will be high (the user will like the item) when the users' factors and the item's factors are significant, and they also match (they have similar values). In Fig. 1 example, we can observe that first and the last factors have similar values: they match each other. The user and the item third factors match, but they are not relevant. Finally,
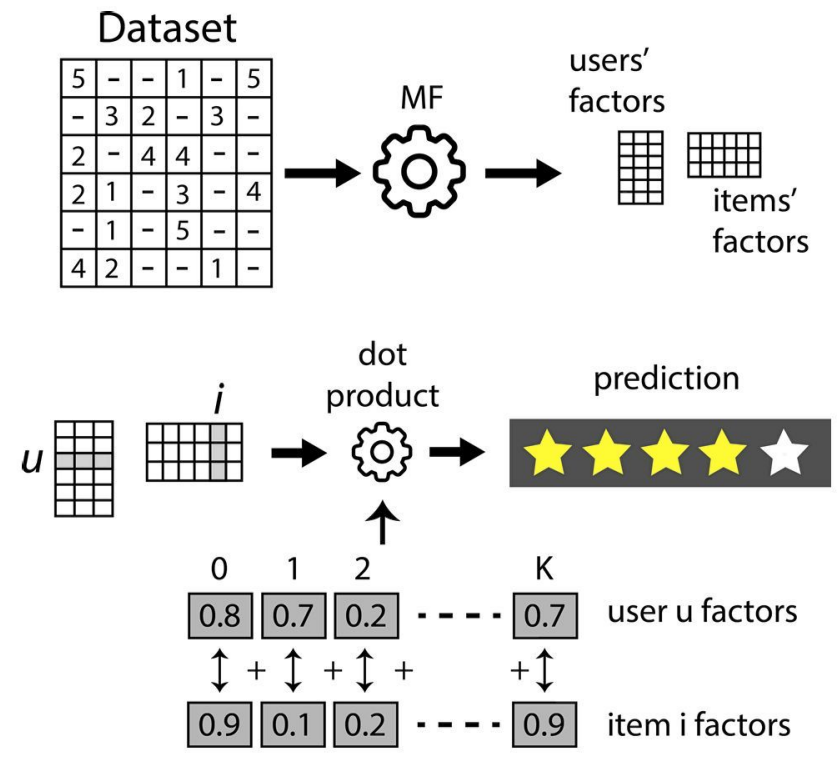

Fig. 1 Matrix factorization and the dot product to make predictions

the second factors do not match. Each user's factor encodes some features combination; a simplistic view could state that the fifth factor encodes: the user is female and young, whereas third factor encodes that she is a female and she likes musical films. Please note that each feature can be coded in several factors. Items are also encoded in $K$ factors; e.g.: Avatar film factor fourth could encode 'young', 'scify' and 'popular', whereas the first factor could encode 'scifi' and 'current'.

It would be great if the MF models could return the semantic meaning of each one of the $K$ chosen factors, but in fact none of the MF models can do it. MF can predict how much a user will like a not voted item, and even it can relate users or items by measuring the distance between their factors, but MF cannot directly establish why it predicts that you will like an item or you will not like another one. MF just acts on the ratings; it does not directly process demographic information (gender, age, etc.) because it has not been designed to do it. To better understanding this concept Fig. 2 shows, in grey colour, the hidden factors, both the items and the users; it means that we do not know the semantics of the hidden factors. It would need some algorithmic process to show us all the MF factors from a different perspectives, in the same way that infrared cameras show our environment. This algorithmic process is represented in Fig. 2 as a magnifier glass. The proposed method in this paper performs the represented magnifier glass function, and it can show the semantic meaning of MF factors in those features we have selected (usually demographic ones).

Extracting semantic information from hidden factors is a challenging task, since the matrix factorization methods are 'blackbox' procedures (this is the reason of the 'hidden' 


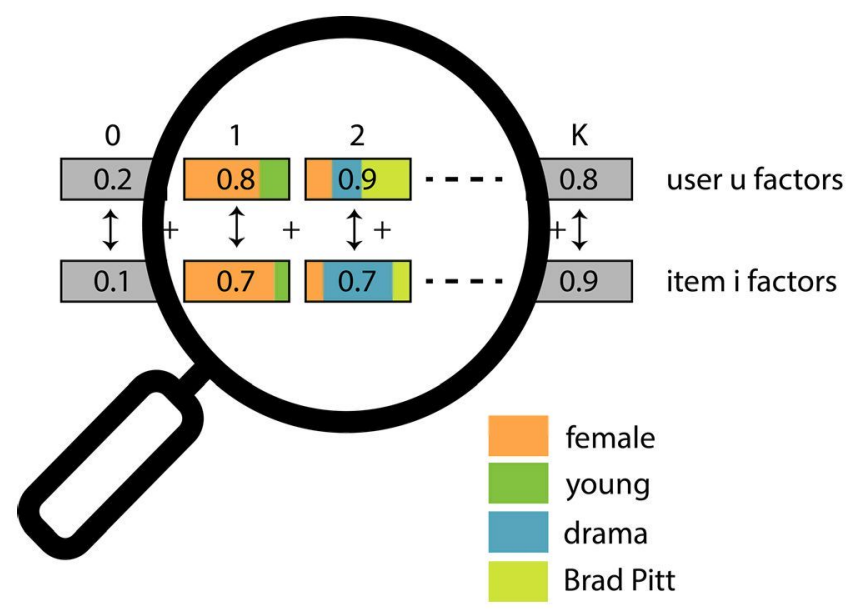

Fig. 2 Matrix factorization hidden factors semantic and applications

term). It is known that each hidden factor can encode several different demographic features. For instance, if we have performed a matrix factorization on a movie-based recommender system dataset, it would be possible that hidden factors $3,5,9$ and 10 code the majority of the users gender demographic information, whereas hidden factors 0 , 2, 3, 7 and 9 code the majority of the users age demographic information. It is not straightforward to isolate the existing demographic features from the obtained hidden factors; it could be made by using statistical methods that relate each user demographic information with each user set of hidden factors, but this approach will only find linear relations between demographic information and hidden factors values. Thus, it is necessary to make use of machine learning algorithms to catch the existing nonlinear relations between demographic data and hidden factors results. In a wide variety of applications, deep learning approaches have been shown to be able to deal with very complex nonlinear relations as long as their number of layers and number of units are adequate to make a model from the data. In this way, we provide a deep learning-based method to unhide demographic information from the existing nonlinear relations between users data and hidden factors values. First of all, it is necessary to understand that this problem can not be modelled as a traditional regression or classification deep learning approach: our starting information is users factors values and users demographic data. We are not predicting demographic values from factors or factors from user values (since the previous matrix factorization made it). Instead, our goal is to extract the nonlinear relations between both pieces of information. There is an image processing field devoted to find the most relevant areas of the input images involved in the classification of their classes (elephants, cats, etc.); its objective is to provide visual explanations from deep networks via gradient-based localization, and its operative is to first

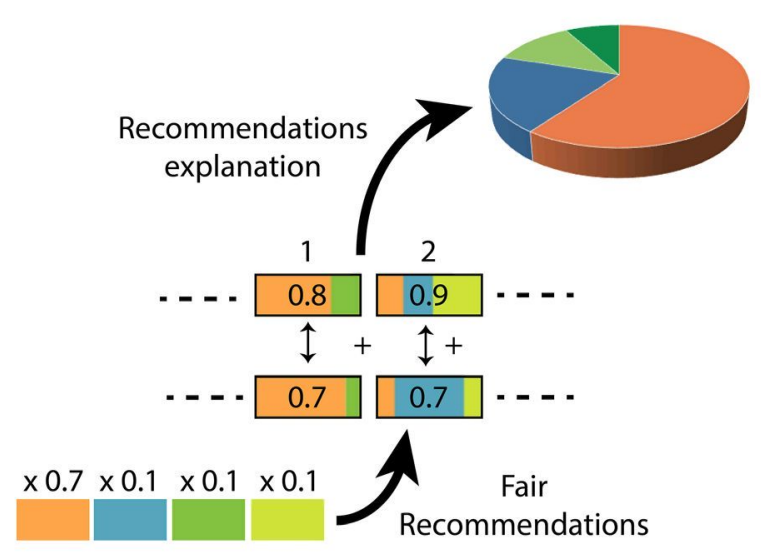

make a classification followed by a backward gradientbased process. Through these two processes it is possible to 'unhide' the complex existing relations between the neural network inputs and outputs by means of a neural network generative process. In this paper, we have adapted this generative process to act in a collaborative filtering context instead of the traditional images one.

Inside the magnifier, it is shown a coloured new information. It tells us about the different degrees of demographic features each factor encodes; e.g.: the user u factor 1 has a big proportion of female feature, followed by a less proportion of young user; in the same way, item i factor 2 mostly encodes a drama film that could like to Brad Pitt's female fans. To get this, additional information is important, since it opens the door to design improved methods in different RS research fields, such as recommendation to groups of users or recommendation to users who share minority preferences. In the former case, a representative virtual user can be obtained by combining the factors values with the factor demographic proportions, in the latter case, a new feature 'minority' can be created to identify minority users. We must realize that current RSs contain minorities of users because different demographic groups are rarely represented in the same proportions and because, beyond demographic issues, it usually exist clusters of users sharing some minority preferences e.g. fans of black and white movies.

Beyond the two previous examples, we have selected two main RS research fields where the proposed method can be particularly important: explanation of recommendations and fair recommendations. The right side of Fig. 2 shows the proposed strategies to address both objectives: recommendations explanation can be based on the impact that each demographic feature has in the recommendation; in this example, we can inform to the user that the recommendation is mostly based on the feminine component 
of the film, and to a much lesser extent because it is a drama film that usually like Brad Pitt's female fans. Fair recommendations can be obtained acting on the dot product stage, by positively weighting the desired demographic features; Fig. 2 shows an example where female factors are privileged with most of the prediction importance: it is an example of fair recommendations applied to the female minority group (most of the RS datasets are biased in gender and age).

The recommendation fairness approach is innovative in the field. Some of the CF fairness research has been focused on the KNN algorithm, since it is a white box approach that allows to design tailored solutions, such in [13] where fairness is obtained by choosing balanced neighbourhoods. In the model-based CF data, biases have been studied as a source of unfair recommendations $[12,16]$. Diversity in recommendations leads to unfair results and discrimination [33], but it is also necessary to balance different goals such as fairness, accuracy, diversity and novelty. Bias disparity has been defined as "how much an individual's recommendation list deviates from his or her original preferences in the training set" [51]. Research in CF fairness has been focused on study the datasets bias rather than to design models to tackle the problem: "teams typically look to their training datasets, not their machine learning models, as the most important place to intervene to improve fairness in their products" [24]. A review of the RS fairness issue is presented in [14], where some frontiers in the field are highlighted. The MF method cannot easily manage the two main sources of imbalanced data: observation bias and population imbalance [58]. As it can be seen, no modelbased $\mathrm{CF}$ approach have been conducted in the same line as the proposed one in this paper; additionally, our method makes use of DL technology, and this is a specific field with little research made in the RS fairness issue: in the DL-based RS survey [39] fairness is not mentioned, not even in its research directions section. In the review paper [4], fairness is not addressed, either.

The recommendation explanations [41] research field has a KNN-based area $[43,60]$ that is not relevant to our model-based approach. Several strategies have been designed to address CF explanations: graphs have been used to relate recommendations sources [36], explanations to group recommendations are also designed based on the group social reality looking for positive reactions from the members of each group [45].

Recommendations have also been explained by using temporal information of the ratings [5, 52]. Trees have been shown where neighbour users and related items are drawn around the recommended user position [23]. As far as we know, there is not a published DL model to address the explanations of $\mathrm{CF}$ recommendations made through $\mathrm{MF}$ factors; nevertheless, there is a paper that emphasizes the importance of the demographic information versus content information in CF explanations [6].

Feature selection is also related to this paper, since we test the proposed method results by selecting the most promising factors, discarding the rest and measuring the impact of the filtering. Figure 3 shows the concept: we can compare the classification results on a demographic target (e.g: gender) by using the $K$ existing factors versus the classification results just using a subset of the $K$ factors (only 2 factors in this example). The more similar the classification accuracy, the best the performed feature selection. To claim the superiority of the DL-proposed feature selection method, we compare it with a set of popular feature selection baselines: logistic [40], entropy [26], variance [55] and principal component analysis (PCA) [29]. Fairness impact can also be tested, since focusing on demographic feature selection we can obtain demographic-based fair recommendations.

Nowadays, DL is a very active research field in which a multitude of applications are being developed in a wide variety of scenarios. A discussion of DL algorithms used in the field of solar and wind energy resources is provided in [49]. Another engineering application of DL is to find the adequate parameter values involved in the existing methods of hydrogen production in order to minimize the necessary energy to perform the process [17]. In addition, DL architectures have proven to be really competitive in the image classification task, by means of Convolutional Neural Network (CNNs). A representative example is shown in [2], where four types of carps (common, grass, bighead and silver) are identified. Additionally, diverse soft computing techniques are used in real-life applications, such as in [57], where several soft computing approaches have been used to rainfall prediction. Prediction of temperature distributions of thermal processes has been addressed in [18] by combining the Karhunen-Loève (KL)

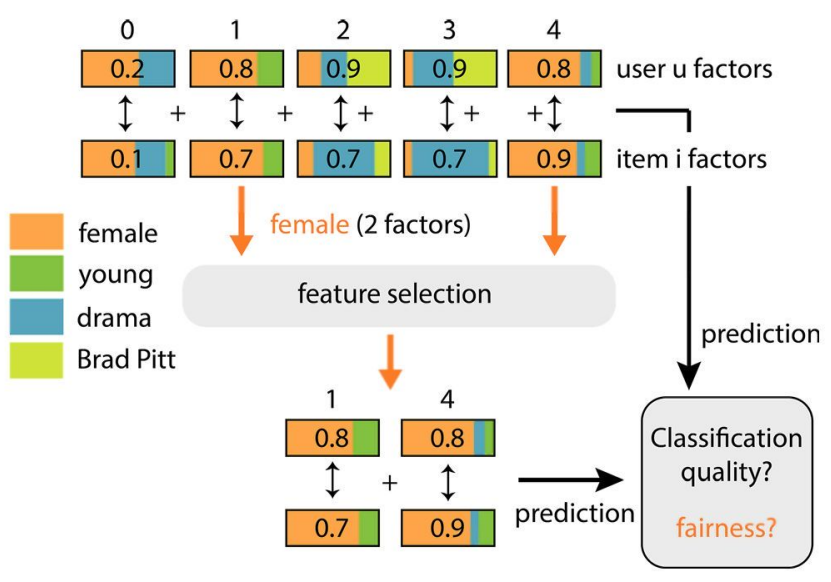

Fig. 3 Feature selection based on demographic information 
decomposition, a multilayer perceptron (MLP), and a long short-term memory (LSTM) network. Finally, the swarm optimization firefly algorithm has been improved by using dimensionally Cauchy mutation [54] .

Finally, there is a DL research field we have borrowed from the image processing area to act as a kernel of the proposed method: the deep networks gradient-based localization. The gradient-based localization algorithm has been selected since it is the most successful current approach to tackle the unhiding problem in the image processing area. It is a sophisticated method that takes advantage of both the backpropagation learning model and the feedforward stage to find the most relevant areas in the neural network activation maps. Other feasible alternatives in the CF area are: (a) statistical correlation between demographic and hidden factors, and (b) machine learning methods to make this task. Statistical methods do not capture the existing nonlinear relations between demographic and factor values, whereas machine learning algorithms do not reach the deep learning capacities to act on nonlinear relations.

To illustrate this idea, let us focus on some examples. Grad-Cam [48] uses any target concept (say 'cat') in a classification network to generate a localization map. It activates the relevant areas, in the image, that encode the concept. Grad-Cam generalizes the Class Activation Mapping (CAM) research [61], where generic localizable DL representations are built. CAM uses the global average pooling as a structural regularizer [35]. Neural Style Transfer (NST) [28] is also a reference to the proposed approach; in this case, a source image is converted to the style of another image that acts as a target. This is made by minimizing the gradient between the source image and one or several chosen filters of a CNN. Our method performs this operation, using a noisy source instead of a regular image. The NST was introduced by Gatys et al. [20] using intermediate layers of the Visual Geometry Group (VGG) 19 [56] network to catch different styles. The style representation has been based on the Gram matrix [19] by matching style and stylized images. The NST implements a particular realization of the above-mentioned gradientbased localization algorithm. In this case, the underlying relation between an input image and a learnt map activation style is used to find the input image that best fits with the map activation style. The main advantage of adopting this approach is the use of the neural network learnt model in order to iteratively (gradient localization) refine the input image from a random picture to the image that fits both with the original picture and the map activation style. As mentioned in the gradient-based localization algorithm discussion, the feasible alternatives to the proposed method are: statistical approaches and the machine learning algorithms. None of them are able to accurately catch the nonlinear relations existing in both the image and the RS contexts.

To graphically show the concept, we have designed an NST and fed it with two Picasso's pieces of art; as it can be seen in Fig. 4 , the style image has been passed to the source image. We have chosen the 'block1_pool' and 'block2_pool' layers of the VGG-19 network as style filters.

The rest of the paper has been structured as follows: in Sect. 2 the proposed method is explained, and the experiments design is defined. Section 3 shows the experiments' results and their discussions. Finally, Sect. 4 contains the main conclusions of the paper and the future works.

\section{Model}

The proposed method to unhide MF factors is inspired in the gradient-based localization [20] and the neural transfer learning [56] techniques. Since it is a DL approach to unhide factors, we have called it DeepUnHide. First, we must design an architecture and then to apply the proposed method to it. Figure 5 shows the DeepUnHide architecture; it is composed of three abstraction levels: raw data, Machine Learning (ML) and DL. The raw data abstraction level feeds the architecture with the necessary information, in our case, it just needs the CF matrix of ratings and the selected demographic information (gender, age, etc.). The ML abstraction level is in charge of providing the MF hidden factors. For that purpose, in the proposed model, we used standard MF methods as PMF [38]. PMF is a representative MF method since it can be considered as a MF baseline. We have chosen it to ease the reproducibility of the experiments described in this paper and since it is considered as the de facto standard. As mentioned, there is a range of $\mathrm{MF}$ alternatives such as $\mathrm{NMF}$ and Bayesian nonnegative matrix factorization (BNMF). Recommendation accuracy may vary using different MF methods, but it is not particularly relevant to this paper objective, since all the MF methods share the same data reduction design and all of them provide factors that are hidden. Moreover, all of them code demographic information in a nonlinear way.

As it can be seen in Fig. 5, we will only take the users' factors, since we want to explain recommendations based

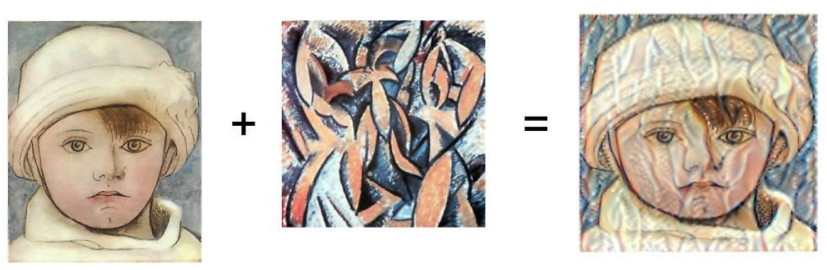

Fig. 4 Example of neural style transfer 


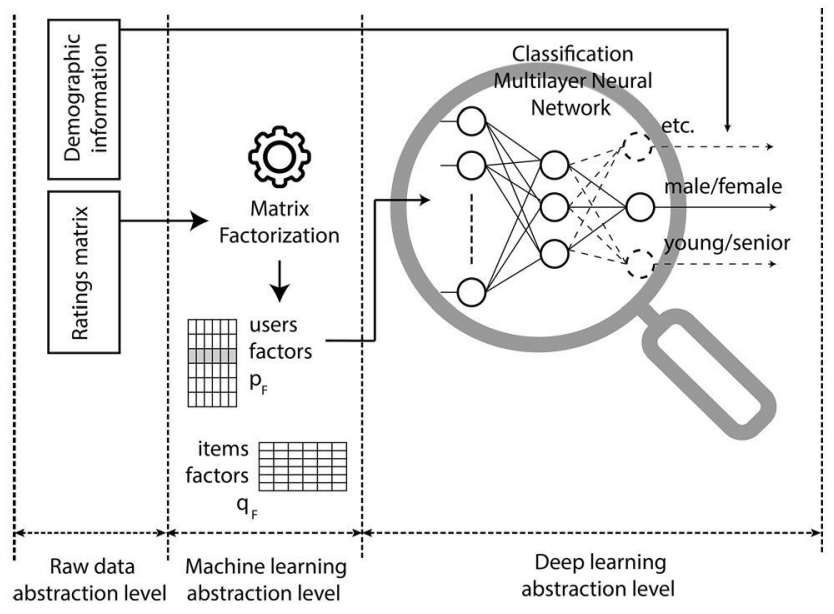

Fig. 5 DeepUnHide architecture

on demographic data related with users. It is also possible to explain recommendations based on demographic data related to items (genre, popularity, director, etc.), in this case, we would take the items' factors to feed the following architectural layer. Our last abstraction level is the DL one: we make use of a multilayer neural network (MLN) to classify users by demographic information. In this paper, as an example, we have chosen the male/female and young/ senior groups. Notice that our objective is not to classify users: We train this MLN to feed the proposed method with the learned weights of the neural network.

\subsection{Gradient localization for image processing}

Once the architecture is set, we can explain the details of the proposed DeepUnHide method, in which we will use the MLN information shown with the magnifier glass metaphor in Fig. 5. In the gradient-based localization [20], conceptually we make a process similar to the one shown in Fig. 4, but there are some key differences; one of them is that we do not have a defined source image: we will use a noise source. Another difference is that instead of using images, our source is a list of hidden factors. To graphically explain the first concept, we make use of the image processing field: Fig. 6 visually shows the learnt pattern of each filter in the VGG-16 Block4_conv1 layer. These filters help to classify some of the images used to train the VGG-16 CNN [30]. Each one of these map activations can serve to get the input pattern that best active the corresponding filter: this is the gradient-based localization key. To obtain the mentioned input patterns, we apply an initial random noise image to the input of the classification neural network. Afterwards, we make use of the gradient descent algorithm to iteratively change the input image until the loss function is minimized. Here, the loss function is the distance between each activation map the input image

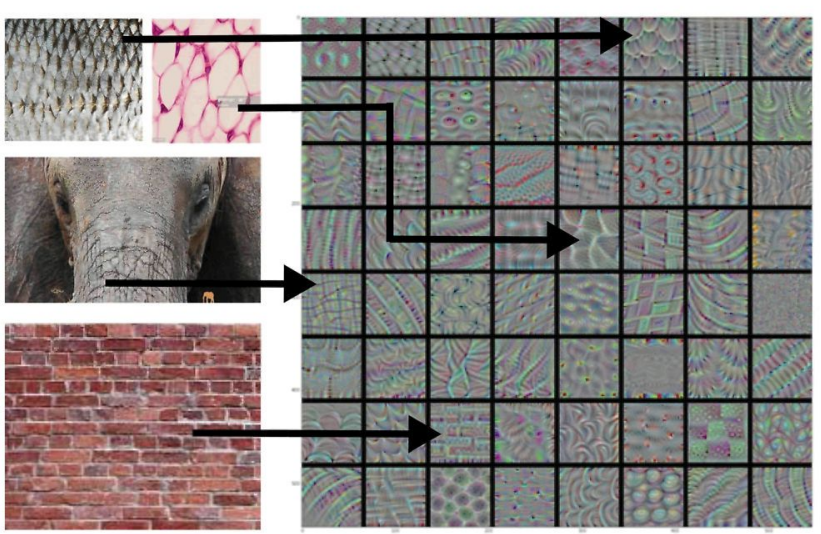

Fig. 6 Learnt patterns in the 64 filters of the VGG-16 Block4_conv1 layer

generates and the chosen filter values. Since we are trying to minimize the difference between each activation map and the input image, any suitable distance metric can be used to implement the loss function. Mean squared differences (MSD) is usually chosen to accomplish this task in the neural style transfer context [28]. Moreover, the gradient descent algorithm can be optimized in different ways, such as the stochastic gradient descent, the Hessian approximation, and the Levenberg-Marquardt algorithm. Adopting these algorithms will not improve results in the proposed method, beyond a convergence speed increase. However, alternative minimization methods could be proposed to find the input image that better fits the image style: genetic algorithms, particle swarm optimization, pattern search, etc. The use of these models is rare in the generative field and thus they are not considered as alternative in our research.

It is worthy to mention that random noise is typically used in NST [28], since it helps to avoid overfitting. In fact, the use of random noise can be considered equivalent to Tikhonov Regularization [7]. As stated in [46], "Heuristically, we might expect that the noise will 'smear out' each data point and make it difficult for the network to fit individual data points precisely, and hence will reduce over-fitting. In practice, it has been demonstrated that training with noise can indeed lead to improvements in network generalization". As an alternative, in the image context, an input sample with the expected pattern could be provided to speed up the training process, with the risk of making it difficult to obtain the expected solution. However, in the RS context, we can not provide a reasonable set of initial factor values, since we know nothing at all about the distribution of the demographic information.

Each row in Fig. 7 shows an example of the aforementioned process: rows contain the gradient descent result of applying a noisy image (left) to the $\mathrm{CNN}$, by minimizing the loss differences with four of the filters in Fig. 6. Each 

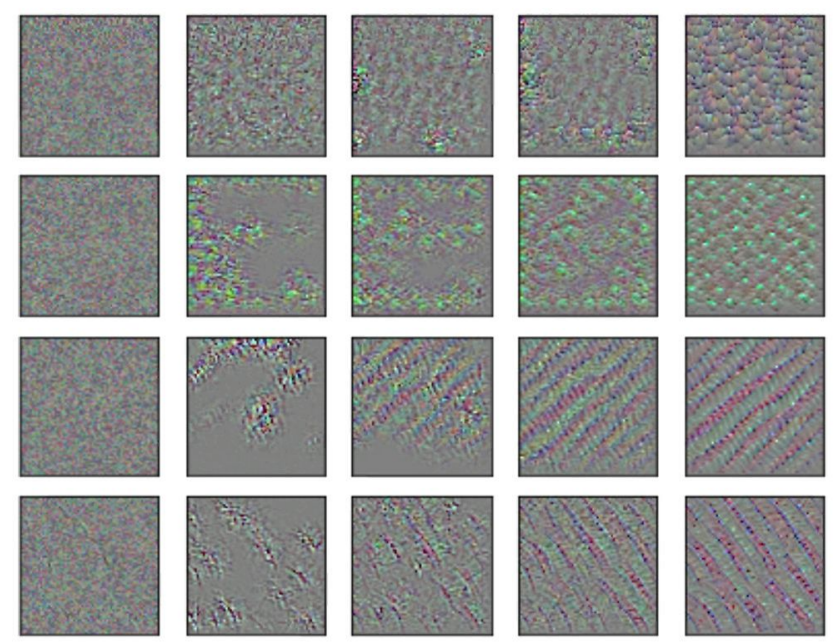

Fig. 7 Gradient descent intermediate images obtained from a noisy picture to four of the activation maps in Fig. 6

of the right-most images of Fig. 7 shows the input pattern that maximizes its corresponding filter detection. They can be considered as representative patterns in some areas of different types of images. What is relevant to us is the concept that using gradient descent on a pre-trained MLN, we can find representative input patterns of the output targets. This is a key concept in the generative learning field $[19,20]$. Moving to the RS field and using the DeepUnHide architecture (Fig. 5), we can find representative patterns of demographic features or, more precisely, we can find the user factors values that best represent the male, female, young, senior, etc. users. Observe that we expect to find demographic information in the users' factors since the hidden factors have been obtained in a dimensionality reduction process, in the same way that the map activations in the $\mathrm{CNN}$ context. In both cases we are extracting valuable information from hidden factors by using the same gradient-based localization algorithm.

\subsection{Gradient localization DeepUnHide}

The proposed DeepUnHide method is explained in Fig. 8. Starting from the trained MLN in the DeepUnHide architecture (Fig. 5), an initial random list of factors, or an initial list of factors filled to 0 are presented to the MLN ("initial list of noisy factors"). Using this starting vector, a feed forward process is conducted to obtain the prediction; then an output and a loss error are obtained; e.g.: we expect the 0 value in the female case or the 1 value in the male one. Then, the gradient descent algorithm obtains the input values that minimize the error, in the first iteration. As usual in the gradient descent operative, the process is repeated until the error reaches a threshold or until a prefixed number of iterations have been run. At the end of the

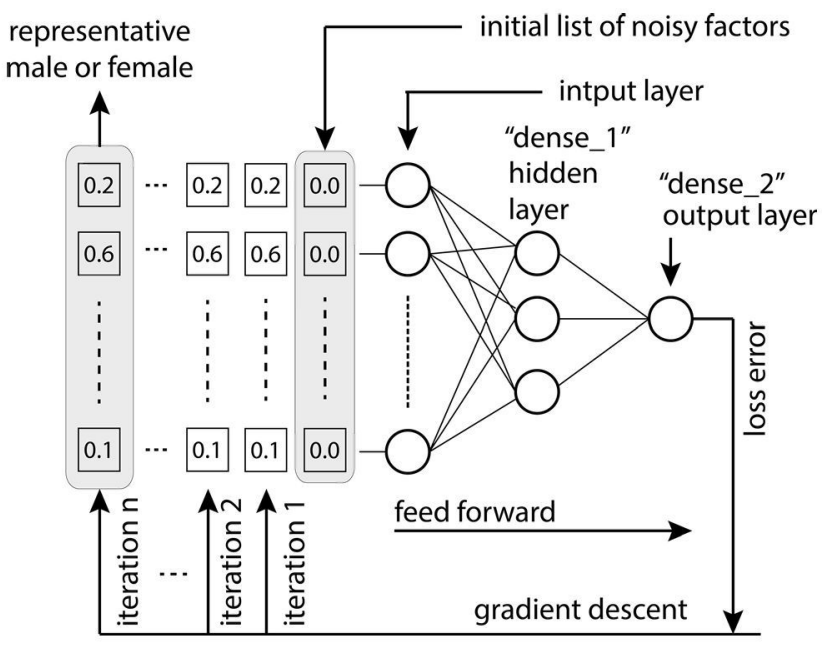

Fig. 8 DeepUnHide gradient-based localization method

process, we get the factors values of the representative demographic user (male, female, young, etc.). Please note that repeating this process for a set of demographic features we can obtain the proportions shown in Figs. 2 and 3; it demographically unhides the users' factors. This process can also be done to demographically unhide the items' factors, since items' and users' hidden factors share the same vector space for codification.

\subsection{Mathematical formulation of DeepUnHide}

To be precise, suppose that we have an initial spare matrix of ratings $R=\left(r_{u, i}\right)$, where $r_{u, i}$ is the rating that user $u$ assigned to item $i$ (say, in a discrete scale from 0 to $N$ ). Denote the number of users in the model by $U$ and the number of items by $I$ so that $R$ is a $U \times I$ matrix.

The objective of PMF is to find a dense matrix $\tilde{R}$ that coincides with $R$ as much as possible in the known ratings. For this purpose, we look for a factorization of the form $\tilde{R}=P Q^{t}$ where $P$ is a $U \times K$ matrix and $Q$ is a $I \times K$ matrix. The interpretation of these matrices is that the $u$-th row of $P, p_{u}$, is the $K$-dimensional vector of hidden factors of the user $u$; and analogously for the $i$-th row of $Q, q_{i}$, for the hidden factors of the item $i$. In this way, we want to minimize the cost function

$$
\|R-\tilde{R}\|_{2}^{2}=\|R-P Q\|_{2}^{2}=\sum_{r_{u, i} \neq \cdot}\left(r_{u, i}-p_{u} \cdot q_{i}\right)^{2},
$$

where $r_{u, i} \neq$. denotes that the rating of the user $u$ to the item $i$ is known, and $\|\cdot\|_{2}$ denotes the usual euclidean distance between the vectors of known ratings. A standard gradient descent algorithm with regularization for minimizing the cost function leads to the update rule 


$$
\begin{aligned}
& p_{u} \leftarrow p_{u}+\gamma\left(\sum_{r_{u, i} \neq}\left(r_{u, i}-p_{u} \cdot q_{i}\right) q_{i}-\lambda p_{u}\right), \\
& q_{i} \leftarrow q_{i}+\gamma\left(\sum_{r_{u, i} \neq \cdot}\left(r_{u, i}-p_{u} \cdot q_{i}\right) p_{u}-\lambda q_{i}\right) .
\end{aligned}
$$

Here, $\gamma, \lambda>0$ are two hyperparameters of the training method (the steps of the gradient descent).

As mentioned above, in this paper, we will focus on unhiding the users factors, $p_{u}$, so we pull apart the items factors $q_{i}$. Now, we focus on some demographic binary classification into majority/minority group (say male/female or young/senior). For that purpose, we consider a MLN,

$h: \mathbb{R}^{K} \rightarrow \mathbb{R}$,

trying to fit the perfect classification given by $h\left(p_{u}\right)=1$ if $u$ belongs to the majority group and $h\left(p_{u}\right)=0$ if $u$ belongs to the minority group. The neural network $h$ is trained with the usual gradient descent optimization on its parameters (the so-called backpropagation method).

Once this DL step is completed, we look for the factors that maximize the expectancy of $h$ of predicting a given demographic group. Hence, we fix an objective target $t=$ $1,0(t=1$ if we are focusing on the majority group and $t=0$ if we are interested in the minority group). Now, we define the cost function

$$
\mathscr{F}_{t}: \mathbb{R}^{K} \rightarrow \mathbb{R}_{\geq 0}, \quad \mathscr{F}_{t}(p)=\frac{1}{2}(t-h(p))^{2} .
$$

That is, $\mathscr{F}_{t}(p)=0$ if and only if $h(p)=t$, which means that $p \in \mathbb{R}^{K}$ is the 'archetypal' user factors of a member of the demographic group $t$. In order to minimize $\mathscr{F}_{t}$, we use a standard gradient descent algorithm. For this purpose, observe that the gradient of $\mathscr{F F}_{t}$ is given by

$$
\nabla \mathscr{F}_{t}(p)=-(t-h(p)) \nabla h(p) .
$$

Observe that the gradient $\nabla h(p)$ can be easily computed in terms of the internal weights of the MLN by means of the usual backpropagation method. Therefore, the usual gradient descent method leads to the update rule

$p \leftarrow p+\eta(t-h(p)) \nabla h(p)$.

Here, $\eta>0$ is a hyperparameter of the training process that corresponds to the step of the gradient descent. The initial guess for $p$ can be taken as a random vector drawn from a uniform distribution, or just as the zero vector. This process is the so-called gradient localization in the image processing literature.

As a result of this optimization step, we get two preferred user factors $p_{M}, p_{m} \in \mathbb{R}^{K}$ for the majority and the minority group. As mentioned above, these can be understood as the factors of a representative user of each demographic group.
Let us we write the components of these vectors as $p_{M}=$ $\left(p_{M}^{1}, p_{M}^{2}, \ldots, p_{M}^{K}\right)$ and $p_{m}=\left(p_{m}^{1}, p_{m}^{2}, \ldots, p_{m}^{K}\right)$. In order to interpret these vectors as amount of affinity, we normalize them to take values in the interval $[0,1]$ as

$$
\begin{aligned}
& \tilde{p}_{M}=\frac{1}{p_{M}^{\max }-p_{M}^{\min }}\left(p_{M}^{1}-p_{M}^{\min }, \ldots, p_{M}^{K}-p_{M}^{\min }\right), \\
& \tilde{p}_{m}=\frac{1}{p_{m}^{\max }-p_{m}^{\min }}\left(p_{m}^{1}-p_{m}^{\min }, \ldots, p_{m}^{K}-p_{m}^{\min }\right),
\end{aligned}
$$

where $p_{M}^{\max }=\max _{j} p_{M}^{j}, \quad p_{M}^{\min }=\min _{j} p_{M}^{j}, \quad p_{m}^{\max }=\max _{j} p_{m}^{j}$ and $p_{m}^{\min }=\min _{j} p_{m}^{j}$. In this way, a value of $\tilde{p}_{M}^{j}$ (resp. $\tilde{p}_{m}^{j}$ ) near to 1 shows that the $j$-th factor characterizes a hidden characteristic that is like-minded to the majority (resp. minority) group whereas a value near to 0 evidences that the $j$-th factor measures a characteristic that is typically disliked by the majority (resp. minority) group.

This idea leads to a feature selection criterion of relevant factors for the majority (resp. minority) group by sorting the factors $j=1, \ldots, K$ by decreasing value of $\vec{p}_{M}^{j}$ (resp. $\left.\tilde{p}_{m}^{j}\right)$. In this way, fixed a number of desired factors $N<K$, we can obtain the subsets $K_{M}$ and $K_{m}$ of the most relevant $N$ factors for the majority and minority group, respectively.

Moreover, this information can also be used for proving an absolute measure of the importance of each factor to the dichotomy majority/minority, as the distance of this factor between the majority archetypal user and the minority archetypal user. Hence, we take

$$
\operatorname{relevancy}(j)=\left|\tilde{p}_{M}^{j}-\tilde{p}_{m}^{j}\right| \text {. }
$$

In this way, high values of relevancy $(j)$ evidences that the $j$ th factor has typically a large variation from a demographic group to another (say, it is high in the majority group and low in the minority group, or vice versa), whereas low values of relevancy $(j)$ point out that this factor is similar in both demographic groups. Therefore, factors with high relevancy are the best indicators of the membership of an user to a group. Again, this relevancy can also be used as a feature selection criterion for choosing the factors that are more relevant for the associated classification problem.

\subsection{Implementation of the model}

DeepUnHide has been implemented using Keras and Python. Other serious alternatives to Keras could be considered as the NVIDIA Compute Unified Device Architecture (CUDA), Deeplearning4j, the MIT-licenced deep learning framework DeepPy, the machine learning ScikitLearn, TensorFlow, the python library Theano, and the open source machine learning library Torch. ScikitLearn has not been chosen due to its poor support for neural networks, Deeplearning $4 j$ is a Java API, TensorFlow is more flexible than Keras, but we do not need to exploit 
such flexibility. Keras, CUDA, DeepPy and Theano could adequately be used to implement this research; Keras has been chosen since it offers rich and simple APIs, it can be used in an easy way. In addition, it is also very popular nowadays: Keras is the most used deep learning framework among top-5 winning teams on Kaggle, it can scale to large clusters of GPUs and it takes advantage of the full deployment capabilities of the TensorFlow platform.

Algorithm 1 shows the internals of the proposed method. Since it is a really short piece of code, it has been considered useful to include the algorithm in this paper in order to explain the method, to easily reproduce the experiments and to base some future works on it. Previous to running the shown procedure, we have trained a MLN using Keras. In our example, we have chosen an architecture with 2 layers, being 'dense_1' the hidden layer and 'dense_2' the output layer (see Fig. 8). Line 2 establishes the output of the model: "dense_2" layer, in the neural network drawn in Fig. 8. Line 3 sets the loss function: in our case, the MLN correctly predicts demographic features. Line 4 makes the hard work, obtaining the gradients of the input with regard to the loss. Line 5 just normalizes the gradients. Line 6 returns the loss and the gradient obtained from the input (input factors). These input factors are initialized in line 7 . Then, a gradient descent loop is set, in line 8 , to run each established iteration and to obtain the new gradient values, in line 9. Finally, the input factors are updated in little 0.1 steps, in line 10. The 0.1 step is the usual value chosen for image gradient-based localization. We have tested smaller values in the RS context, obtaining similar results and slower response times. This line 10 of code generates the subsequent input factors shown on the left of Fig. 8 , where the result is shown in grey background.

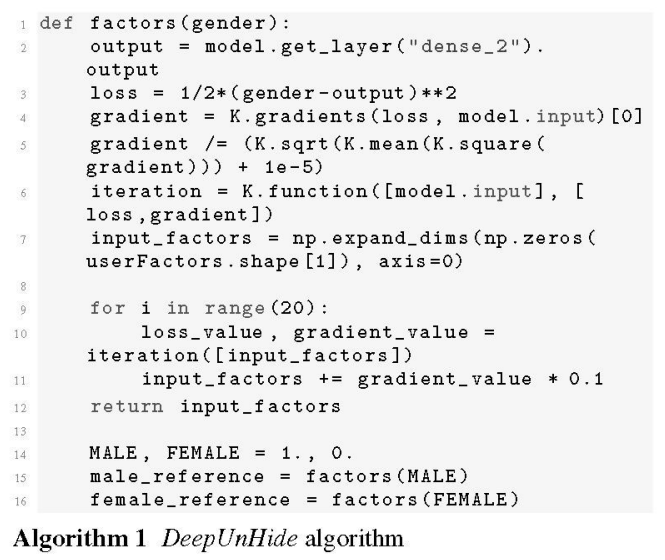

\section{Experiments and results}

This section tests the proposed DL method and architecture on two representative and public datasets. Four consolidated feature selection baselines are used: logistic, entropy, variance and PCA. They were chosen since they represent four different approaches in ML for dealing with feature selection problems. Others feature selection methods can be considered, like minimum-redundancy-maximum-relevance (mRMR) feature selection [44], correlation-based methods [21] or consistency-based approaches [15] but, in a way or another, all of them belong to one of the four classes represented by the chosen methods.

From a purely statistical point of view, variance is a natural criterion for filtering out irrelevant features: the larger the variance, the wider its confidence intervals, so a large variance of a feature means that each measurement contains lot of information since the expected value is "uncertain". In other words, features with low variance are not informative since their particular value are just small noisy perturbations of a constant. Related with this concept, but from a different point of view is the entropy criterion that measures the amount of information that a feature provides, understood as amount of "surprise". This is a widely used criterion for evaluating informativeness, not only in feature selection, but also in other ML methods like construction of decision trees. In contrast with these quality-based methods for feature selection, the PCA algorithm is also used in these experiments, providing feature selection tools from a different point of view, namely, of topological-geometric nature according to dimensionally reduction criteria. Finally, a model-based approach was applied like feature selection via logistic regression. In sharp contrast with the previous methods, this is a post hoc approach that seeks to maximize feature informativeness for an already solved classification problem. For this purpose, the feature selection criterion uses the explainability of the model to unravel the most important features. Notice that the choice of the particular classification method is secondary here: we are focusing on the quality of the features, as extracted by the method, not on the output of the method itself. For this reason, relatively small variations in the performance of the classification method are irrelevant for the choice of the most informative features, which are expected not to vary between methods. For this reason, logistic classification was selected for these experiments, since it is a method that joins a clear explainability of the model through its weights, a fast training and a typical avoidance of overfitting thanks to its simplicity.

The classification accuracy score has been selected to measure the quality of the results. Notice that, since we are 
not predicting ratings, the typical quality prediction measures (MAE, RMSE, etc.) have not sense in this context. The same happens with the standard CF quality recommendation measures (precision, recall, F1, etc.). On the contrary, we are testing whether our method correctly classifies samples in their corresponding demographic classes (e.g. gender and age) or not. For this reason, the experiments are focused on measuring the classification accuracy in the $[0,1]$ interval.

Most of the experiments compare the results obtained by choosing different numbers of selected features. Four classification models are used in the experiments: neural networks, logistic regression, support vector machine (SVM) and random forest. The combination of these four classification models represents most of the classification cases that are carried out in data science. They are representative of the state-of-art: neural networks perform very complex classifications, particularly when spatial or temporal structure of data occurs (images, speech, noisy data, etc.); random forest is a decision tree-based ensemble that usually obtains the best accuracy among the machine learning methods; SVM is a very fast and accurate classification method that gives rise to good results when classes are not deeply intermingled, even if the decision boundary is highly nonlinear; finally, logistic regression is a representant of the simplest classification ML methods, quick and straightforward but typically providing quite good solutions thanks to its avoidance of overfitting. Bayesian classification methods have not been considered since research does not state adequate results neither in image processing nor in $\mathrm{CF}$.

Cross validation has been implemented by using a $70 \%$ training set, a $10 \%$ validation set and a $20 \%$ testing set. This is a typical set of validation parameter values when datasets have an adequate size, such as in our case. The chosen datasets to make the experiments are the popular MovieLens and the MyAnimeList. Both of them contain demographic information. Although there is a large number of available open RS datasets, very few ones contain valuable demographic information. Some of the datasets provide social information and some others provide context aware information e.g. Quality of service (QoS) parameters, global positioning system (GPS) coordinates, etc. There are available e-commerce datasets containing ratings and products reviews, datasets that only contain rating values, datasets with incomplete demographic information, etc. Discarding inadequate $\mathrm{CF}$ datasets for our purpose of focusing on demographic data, and selecting the more representative ones, we have chosen the aforementioned MovieLens and MyAnimeList datasets to perform the designed experiments.

MyAnimeList contains more than five million of ratings, and the selected MovieLens version has only 100,000
Table 1 Datasets used in the experimentation

\begin{tabular}{lrlrl}
\hline & \#users & \#items & \multicolumn{1}{c}{ \#ratings } & Scores \\
\hline MovieLens & 943 & 1682 & 100,000 & $1-5$ \\
MyAnimeList & 69,600 & 9927 & $5,788,207$ & $1-10$ \\
\hline
\end{tabular}

ratings; in this way, we will test the proposed method on two datasets with very different sizes. Some relevant dataset facts are shown in Table 1 . We will show all the results from the MovieLens dataset, and the more representative ones from the MyAnimeList dataset. Two demographic features have been tested: gender and age, founding little differences in their results. Gender and age have been chosen because they are relevant demographic factors: they are representative and they are common demographic fields to both the MovieLens and MyAnimeList selected datasets. Some other demographic features, such as the zip code, are not representative (to the specific CF context) or they are not available in most of the open datasets. To keep the paper in a reasonable size, and to avoid including redundant information, figures in this section are restricted to the gender results.

Throughout the performed experiments, MF has been processed by using a large number of factors, namely $K=100$. Observe that customary values for $K$ range from 20 to 30 in the CF MF processes in order to obtain a suitable prediction accuracy. Nevertheless, our goal is not just to preserve accuracy: we want to isolate demographic information from factor values. For this reason, it is important to provide a large number $K$ of factors making it possible to spread demographic information among factors and avoiding excessive concentrations of demographic data coded in a small number of factors.

Once the MF has been run on both datasets, the following step is to train the MLN included in the DeepUnHide Architecture (Fig. 5). We have classified users for both the gender and the age demographic features. The age groups are under 40 years old and 40 or more years old. Figure 9 shows the classification accuracy obtained in the MovieLens dataset for both the gender and the age demographic features. Regarding the MyAnimeList dataset, it reaches a 0.82 gender accuracy. The designed MLN for MovieLens contains a 100 neurons input layer, a 10 neurons hidden layer with Rectified Linear Unit (ReLU) activation, a 0.3 dropout layer, and finally, the 2 neurons output layer to encode gender and age, using sigmoid activation. The chosen loss function is binary cross-entropy, and the optimizer is RMSprop. In the case of the MyAnimeList dataset the used MLN is similar to the MovieLens one, with the only difference that the hidden layer contains 20 neurons. The number of hidden neurons, both in the MovieLens and MyAnime datasets, have been 


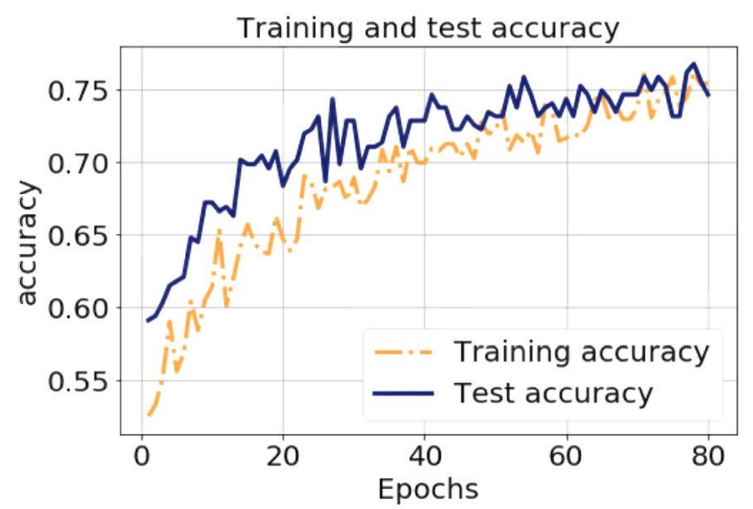

(a)

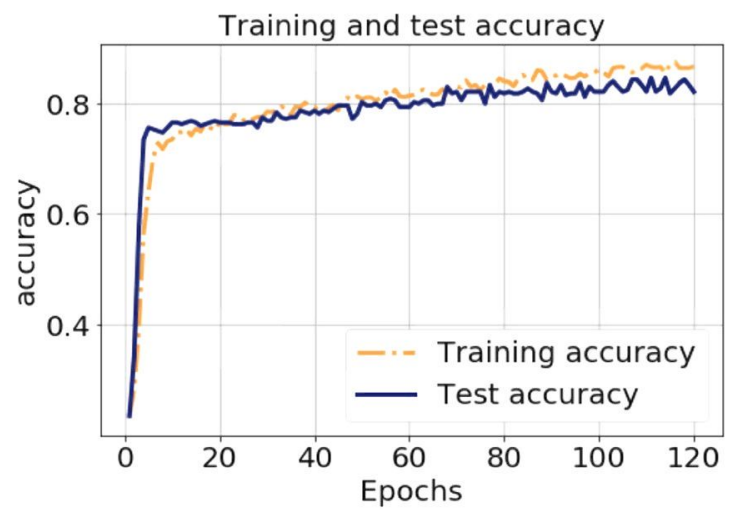

(b)

Fig. 9 Training and test classification accuracy reached in the MLN of the DeepUnHide architecture. Gender classification (a), age classification (b). MovieLens dataset

empirically chosen by running a set of validation experiments. The dropout value 0.3 provides adequate generalization results in both cases.

Once the DeepUnHide architecture MLN (Fig. 5) has been trained, we run DeepUnHide (Fig. 6) to obtain the demographic proportions of each user factor, as seen in Fig. 2. MovieLens gender (male, female) results are shown in Fig. 10: its top graph draws the male (blue) and the female (red) proportions that each factor encodes ( $x$-axis: factors). Representative factors are those which mostly encode male or mostly encode female: it helps to make a feature selection. Middle graph in Fig. 10 shows the normalized absolute difference of the female and male proportions: the largest the absolute difference, the better the factor distinguishes the feature. From these values, we can select those whose normalized absolute difference exceed a threshold, obtaining the most relevant factors (selected features).

As an example, the graph in the bottom of Fig. 10 shows the factors that overtake the 0.5 threshold: they are the result of the proposed DeepUnHide feature selection. Making the same process to the age (young, senior) demographic feature, in the MovieLens dataset, we obtain the results shown in the top graph of Fig. 11 . Please note
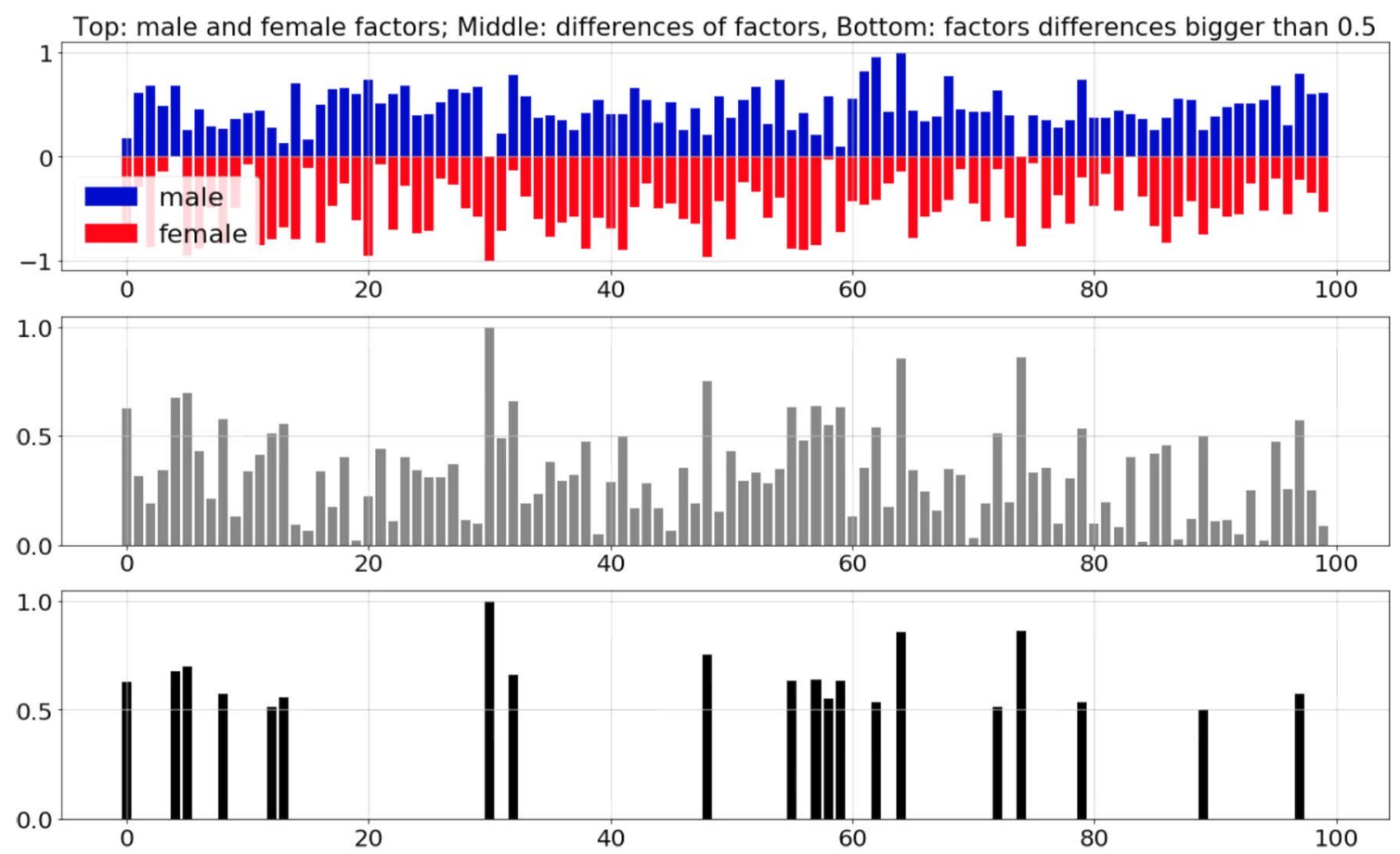

Fig. 10 Male and female proportions encoded in each of the MF factors; MovieLens dataset. $x$-axis: factor number; top graph: male and female proportions; middle graph: normalized absolute difference between male and female proportions; bottom graph: more relevant factors to the gender demographic feature 

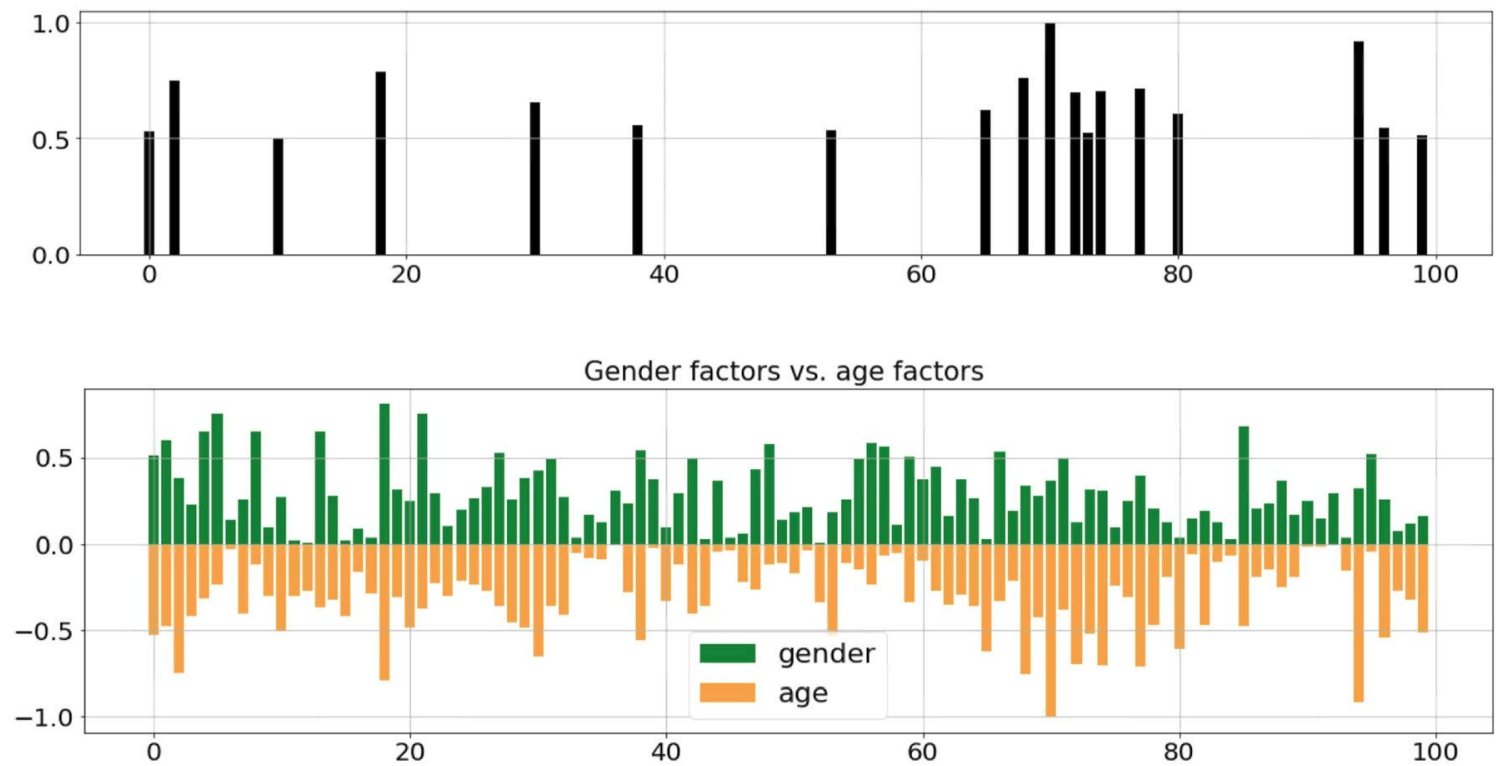

Fig. 11 Top graph: more relevant factors to the age (young, senior) demographic feature; bottom graph: gender versus age proportions for each MF factor. Movielens dataset. $x$-axis: factor number

that the same factor can be relevant to two different demographic features (such as factor 0 in Fig. 10), although it will not be the usual situation when the number of the MF factors is high. Bottom graph in Fig. 11 confronts the proportions of the demographic gender and age features for each MF factor. Explanation of recommendations can be done using this information: since we know each demographic importance for each hidden factor, it is possible to extract this information from the prediction dot product, as shown in Fig. 2 .

Please note that the bottom graph in Fig. 10 shows the set of factors that best discriminate the gender demographic feature, whereas the top graph in Fig. 11 shows the set of factors that best discriminate the age demographic feature. All these factors have been obtained by using a threshold value. Another approach is to select the $N$ factors that best discriminate the desired demographic feature: instead of using the indicated threshold, we just take the $N$ most promising factors. The first two experiments in this section compare the classification quality results obtained by using different $N$ values (different number of factors). These are feature selection experiments; it is expected that the more the $N$ value, the best the quality results. It is also expected that a reduced number of factors can provide accurate classification values. Finally, the proposed DeepUnHide method should show better scores and trends than the baselines do. The first experiment makes use of an $N$ range from 1 to 20; the second experiment uses an $N$ range from 5 to 70; finally, the third experiment fixes $N=50$.

Once the DeepUnHide feature selection is made, we have designed three experiments to test that it is correct and that it improves the state-of-the-art. The three experiments test the classification accuracy quality measure, and all of them compare the proposed approach with several state-of-art feature selection methods: logistic [40], entropy [26], variance [55] and PCA [29]. Additionally, a random baseline is used. Each of the three experiments is explained in detail at a separated subsection.

The first experiment is focused on the four generated users: the representative male, female, young and senior. For this purpose, we carry out a prediction (forward) process on the trained DeepUnHide MLN. Since no training process is involved in the experiment, we can test it by using a reduced number of selected factors. On the other hand, the second experiment conducts several training processes both for the deep proposed method and the logistic, entropy, variance and PCA baselines. The range of the $N$ selected factors must begin with a minimum value which allows the system to store coded results (that we have fixed to 5) and a maximum value to avoid overfitting (that we have fixed to 70 ). This $[5,70]$ range is usual in the RS testing contexts when machine learning processes are involved. Finally, the third experiment involves testing the cartesian product of all the available feature selection methods (DeepUnHide, logistic, entropy, variance and PCA) with all the chosen classification algorithms (logistic, SVM and Random Forest). To avoid an excessive number of results and to make it clear the graph representation, we have selected just one representative value of $N$ that has been fixed to $N=50$, that is, half of the whole available factors. 
Fig. 12 Classification results of the proposed

DeepUnHide method (Deep for short) compared to the feature selection baselines:

logistic [40], entropy [26], variance [55] and PCA [29]. $x$ axis: number of selected factors, $y$-axis: correct classification values ( 0 for female and 1 for male)
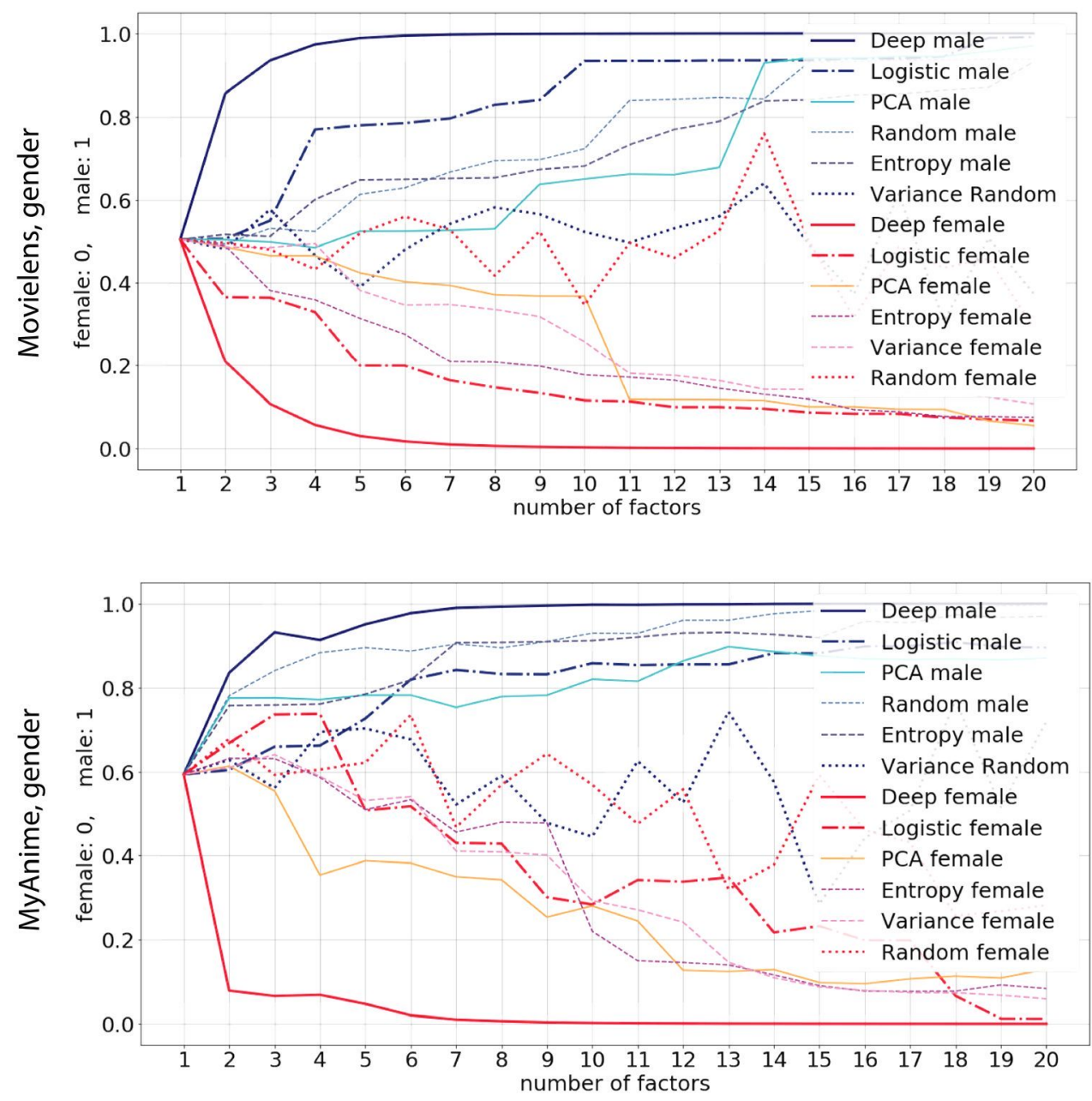

\subsection{Classification of the representative users applying a neural network}

This experiment uses the factors of the representative male and female (Fig. 8, iteration $n$ ). The hypothesis is that correct classification can be achieved by using a reduced set of the selected features. Classification is performed running forward (predicting) the same MLN of the DeepUnHide architecture (Fig. 8). Predictions near to the value 1 can be considered users classified as 'male', whereas predictions near to the value 0 can be considered as 'female' users (same with 'young' and 'senior'). Different classification processes are made using different numbers of factors (from 1 to 20). Fig. 12 shows the obtained results for the tested datasets: MovieLens (top graph) and MyAnimeList (bottom graph) when the gender feature is chosen. The male (blue colour) correct classification value is the number 1 ( $y$-axis) and the female (red colour) correct classification value is the number 0 (y-axis). Both graphs in Fig. 12 show, as expected, that increasing the number of selected factors the classification accuracy raises. Solid lines in Fig. 12 correspond to the proposed DeepUnHide method ("Deep", for short in legends). It can be seen that our method works fine even with a very reduced number of selected factors: using just 3 factors it can correctly classify with small errors. All the baselines need a larger number of factors than the proposed method to reach a similar accuracy; logistic is the best baseline on MovieLens, whereas entropy is better in MyAnimeList. None of them can compete with DeepUnHide. Please note that experiments that selects a very low number of factors (one to three factors) can return an ambiguous classification result. They do not manage enough information to correctly classify users; e.g.: the 0.5 and 0.6 classification values in Fig. 12 , when only one factor is selected. The demographic age classification results are very similar to the gender ones.

\subsection{Classification of all the users applying a neural network}

The previous experiment did not classify all the testing users in the dataset. It just classified the representative male user and the representative female user (by using different numbers of selected factors). Experiments in this section 


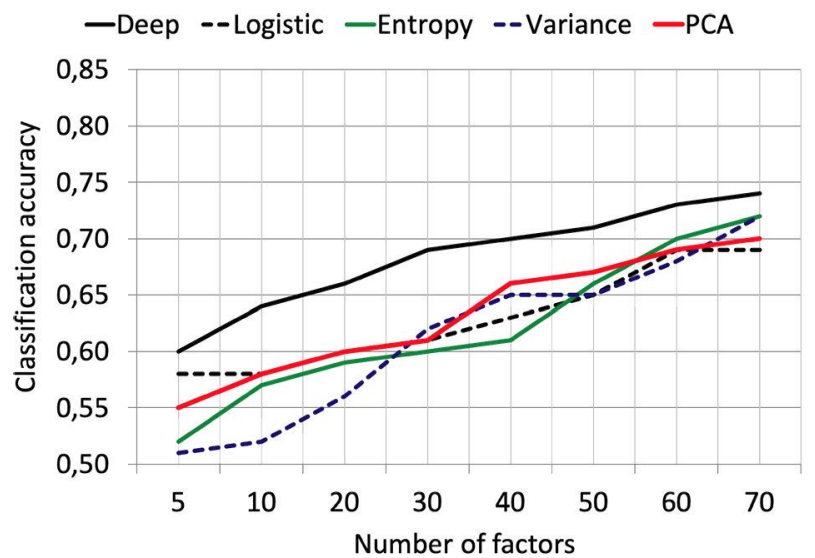

(a)

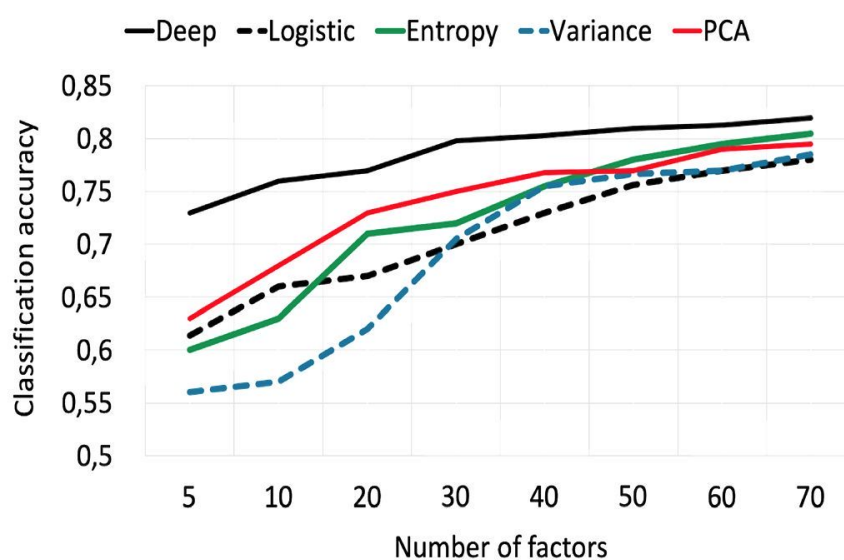

(b)

Fig. 13 Classification accuracy of the users based on their gender; a Movielens, $\mathbf{b}$ MyAnime. $x$-axis: number of selected factors, $y$-axis: accuracy. Proposed method: DeepUnHide (Deep for short). Baselines: logistic [40], entropy [26], variance [55] and PCA [29]

make use of an MLN to classify all the users attending to their gender demographic feature. Hypothesis here is the same than in the previous experiment one: correct classification can be achieved by using a reduced set of selected features. Experiments in this section have been performed varying the number of selected factors. Figure 13 shows the obtained results: as expected, accuracy increases when the number of selected factors grows. The MyAnimeList dataset reaches better classification results; DeepUnHide (Deep for short) improves all the baselines accuracy for all the tested number of selected factors.

\subsection{Classification of all the users applying several machine learning models}

Since the proposed feature selection method and its architecture are based on the DL model, and in the previous section, it was tested the accuracy by means of a DL classifier, it has been considered convenient to make some quality testing based on different classification models. In particular, the ML logistic regression, SVM and random forest have been chosen. In this section, we compare the accuracy score obtained using the mentioned models and applying them to both the proposed DeepUnHide method and the selected baselines. Figure 14 shows the results obtained in the: (a) MovieLens dataset and (b) MyAnimeList dataset. The number of selected factors has been fixed to 50 (half of the whole available factors). In the same line that previous experiments, the proposed Deep feature selection gets better accuracy than the baselines in all the cases. MyAnimeList reaches better classification accuracy than MovieLens, and random forest is the ML model with better results, although they are worse than the DL model (comparing Figs. 13 and 14).

\subsection{Unhiding the hidden factors in the MovieLens dataset}

DeepUnHide has been designed to facilitate the understanding of hidden factors in a MF-based RS by extracting

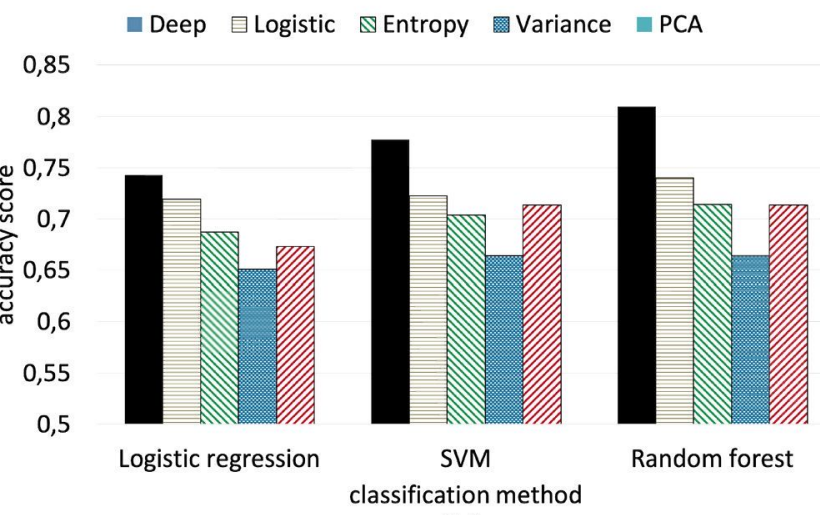

(b)

Fig. 14 Classification accuracy of the users; a Movielens, b MyAnime. $x$-axis: classification model, $y$-axis: accuracy. Proposed feature selection: DeepUnHide (Deep for short). Baselines: logistic [40], entropy [26], variance [55] and PCA [29]. Demographic feature: gender 
demographic information from users. This information can be used to explain the recommendations performed by the RS. It can be done by making use of the hidden factors of the majority archetypal user $p_{M}$ and the minority archetypal user $p_{m}$ (see Sect. 2.3). By selecting their highest hidden factors, we can obtain the most representative factors of the majority group, $K_{M}$, and the minority group, $K_{m}$.

In this experiment, we define an index to assign a minority value to the $i$-th item (say, femininity), $w_{m}(i)$, and equivalently another index to assign a majority value to each item (say, masculinity), $w_{M}(i)$. These two weights can be obtained by comparing the archetypal users $\tilde{p}_{m}$ and $\tilde{p}_{M}$ with the hidden factors of the item $i, q_{i}$. To be precise, we define the minority weight $w_{m}(i)$ of each item $i$ as

$w_{m}(i)=\sum_{j \in K_{m}} \tilde{p}_{m}^{j} \cdot q_{i}^{j}$.

Analogously, we define a majority weight $w_{M}(i)$ of each item $i$ as

$w_{M}(i)=\sum_{j \in K_{M}} \tilde{p}_{M}^{j} \cdot q_{i}^{j}$.

We focus on the MovieLens dataset, by assigning male users to the majority group and female users to the minority group. Using the previously computed archetypal male user, $\tilde{p}_{M}$, and the archetypal female user, $\tilde{p}_{m}$, we compute the coefficients $w_{M}(i)$ and $w_{m}(i)$ for each item of the dataset. The 10 most representative movies of the minority group [i.e. the top 10 items with the highest $w_{m}(i)$ ] are the following:

1. Evita (1996)

2. Crucible, The (1996)

3. Dirty Dancing (1987)

4. Nell (1994)

5. Rosewood (1997)

6. Dante's Peak (1997)

7. Jungle2Jungle (1997)

8. On Golden Pond (1981)

9. My Best Friend's Wedding (1997)

10. Little Women (1994)

On the other hand, the following 10 movies as the most representative of the majority group (i.e. the top 10 items with the highest $w_{M}(i)$ ):

1. Fifth Element, The (1997)

2. Trainspotting (1996)

3. Crumb (1994)

4. Die Hard (1988)

5. Clerks (1994)

6. Aliens (1986)

7. Miller's Crossing (1990)

8. Lost Highway (1997)
9. Brazil (1985)

10. Dances with Wolves (1990)

For this experiment, we have used the top $N=20$ hidden factors of the archetypal users to compute $K_{M}$ and $K_{m}$ and all movies with less than 75 ratings have been filtered out to avoid cold start situations.

\section{Conclusions}

An innovative approach to unhide demographic features in matrix factorization has been presented in this work. It uses the gradient-based localization concept, borrowed from the deep learning image processing. This approach is based on the existing semantic similarities between the MF-dimensional reduction and the deep learning progressive-dimensional reduction. MF encodes the semantic information of the recommender system's users and items inside a common vector space, in the same way as image patters are codified into the internal weight spaces in neural networks. For this reason, the gradient-based localization approach is a flexible solution that should be applicable to other $\mathrm{CF}$ scenarios to unravel the semantic demographic information hidden under the ratings patterns.

In the same way that gradient-based localization creates input patterns that maximize activation maps, in the $\mathrm{CF}$ context, we can find a pattern for each selected demographic feature. As an example: in the same way that we can obtain, via gradient descent localization, a pattern 'elephant skin' that maximizes one of the existing activation maps, we can find, via gradient descent localization, a pattern 'male' that maximizes the male sample classification. In this vein, DeepUnHide is able to obtain patterns of hidden vectors that represent a particular demographic feature. Using this idea, the obtained representative user vector for each demographic feature (say gender) serves to make the feature selection

Overall, the results show an important improvement in the classification accuracy score when the selected features are applied, compared to the baseline methods. It is remarkable the high performance of the obtained demographic factors in the subsequent classification task with pre-trained neural networks, compared with the representative set of features extracted by feature selection baselines (namely logistic, PCA, random, entropy and variance), both in the male and the female demographic cases. As expected, improvements are comparatively higher when the number of chosen factors is low. This means that the proposed DeepUnHide method outperforms the feature selection results of the representative baselines even in the most demanding scenarios. The superiority of the proposed architecture has also been proven when the obtained demographic factors are used to feed an MLN training. The 
results show that the MLN achieve more accurate results when it is trained using the demographic factors obtained through the proposed method, instead of the four feature selection baseline methods. As expected, improvements are higher when the number of chosen factors is low. Finally, several machine learning classification methods (logistic regression, SVM and random forest) have been used instead of the classification MLN. Again, the results show the superiority of the proposed deep feature selection in all the cases: our deep learning approach outperforms baselines in all the chosen representative machine learning and deep learning classification methods.

Therefore, we can assure that the proposed ML method and architecture accurately are able to capture hidden semantic of the MF factors. The obtained results open the door to reach improvements on several representative research fields in the recommender systems area. Recommendation explanation can be addressed by translating from the obtained demographic information to a visual representation of demographic features. Fairness is another important research field where the proposed method has a direct application: fair recommendations can be made by weighting those factors that belong to the biased group of users. The proposed method can also be applied to recommendation of groups of users, making use of the gradient-obtained representative user that can act as virtual user for the group.

In addition, this paper has also some limitations that should be addressed by means of a set of proposed future works. (a) To expand experiments including some other subtler user demographic features such as zip code or salary. (b) To extend experiments including item demographic features such as movie genre or movie premiere, among others. (c) Detection of unreported minority users and/or items, via clustering, for which there is no available demographic information in the dataset in order to study their characteristic features e.g. fans of black and white movies. (d) To expand experiments using some other RS datasets, say of music, e-commerce, etc. (e) Finally, it would be interesting to cluster users or items attending to demographic features. This approach would allow us to obtain a fine grain set of representative hidden factors. Notice that wide groups of demographic-based users or items could not be adequately represented by using a single set of features. For instance, it could be more accurate to cluster male users into several groups and to extract the set of hidden factors that best represent each particular group.

\section{Compliance with ethical standards}

Conflict of interest The authors declare that they have no conflict of interest.

\section{References}

1. Al-Shamri MYH (2016) User profiling approaches for demographic recommender systems. Knowl Based Syst 100:175-187

2. Banan A, Nasiri A, Taheri-Garavand A (2020) Deep learningbased appearance features extraction for automated carp species identification. Aquac Eng 89:102053

3. Barragáns-Martínez AB, Costa-Montenegro E, Burguillo JC, Rey-López M, Mikic-Fonte FA, Peleteiro A (2010) A hybrid content-based and item-based collaborative filtering approach to recommend TV programs enhanced with singular value decomposition. Inf Sci 180(22):4290-4311. https://doi.org/10.1016/j. ins.2010.07.024

4. Batmaz Z, Yurekli A, Bilge A, Kaleli C (2019) A review on deep learning for recommender systems: challenges and remedies. Artif Intell Rev 52(1):1-37

5. Bharadhwaj H, Joshi S (2018) Explanations for temporal recommendations. Künstl Intell 32(4):267-272. https://doi.org/10. 1007/s13218-018-0560-x

6. Bilgic M, Mooney RJ (2005) Explaining recommendations: satisfaction vs. promotion. In: Beyond personalization workshop, IUI, vol 5. p 153

7. Bishop CM (1995) Training with noise is equivalent to Tikhonov regularization. Neural Comput 7(1):108-116

8. Bobadilla J, Alonso S, Hernando A (2020) Deep learning architecture for collaborative filtering recommender systems. Appl Sci $10(7): 2441$

9. Bobadilla J, Ortega F, Gutiérrez A, Alonso S (2020) Classification-based deep neural network architecture for collaborative filtering recommender systems. IJMAI 6(1):68-77. https://doi. org/10.9781/ijimai.2020.02.006

10. Bobadilla J, Ortega F, Hernando A, Gutiérrez A (2013) Recommender systems survey. Knowl Based Syst 46:109-132

11. Bobadilla J, Serradilla F (2020) The effect of sparsity on collaborative filtering metrics. In: Proceedings of the twentieth australasian conference on australasian database - 92. pp 9-18. https://doi.org/10.5555/1862681.1862686

12. Bobadilla J, Lara-Cabrera R, González-Prieto Á, Ortega F (2020) Deepfair: deep learning for improving fairness in recommender systems. Int J Interact Multimed Artif Intell. https://doi.org/10. 9781/ijimai.2020.11.001

13. Burke R, Sonboli N, Ordonez-Gauger A (2018) Balanced neighborhoods for multi-sided fairness in recommendation. In: Friedler SA, Wilson C (eds) Proceedings of the 1st conference on fairness, accountability and transparency, proceedings of machine learning research, vol 81. PMLR, New York, NY, USA, pp 202-214

14. Chouldechova A, Roth A (2020) A snapshot of the frontiers of fairness in machine learning. Commun. ACM 63(5):82-89. https://doi.org/10.1145/3376898

15. Dash M, Liu H (2003) Consistency-based search in feature selection. Artif Intell 151(1-2):155-176

16. Ekstrand MD, Tian M, Kazi MRI, Mehrpouyan H, Kluver D (2020) Exploring author gender in book rating and recommendation. In: Proceedings of the 12th ACM conference on recommender systems. pp 242-250. https://doi.org/10.1145/3240323. 3240373

17. Faizollahzadeh Ardabili S, Najafi B, Shamshirband S, Minaei Bidgoli B, Deo RC, Chau Kw (2018) Computational 
intelligence approach for modeling hydrogen production: a review. Eng Appl. Comput. Fluid Mech. 12(1):438-458

18. Fan Y, Xu K, Wu H, Zheng Y, Tao B (2020) Spatiotemporal modeling for nonlinear distributed thermal processes based on KL decomposition, MLP and LSTM network. IEEE Access $8: 25111-25121$

19. Gatys L, Ecker AS, Bethge M (2015) Texture synthesis using convolutional neural networks. In: Cortes C, Lawrence ND, Lee DD, Sugiyama M, Garnett R (eds) Advances in neural information processing systems 28. Curran Associates, Inc, pp 262-270

20. Gatys LA, Ecker AS, Bethge M (2016) Image style transfer using convolutional neural networks. In: 2016 IEEE conference on computer vision and pattern recognition, CVPR 2016, Las Vegas, NV, USA, June 27-30, 2016. IEEE Computer Society, pp 2414-2423. https://doi.org/10.1109/CVPR.2016.265

21. Hall MA (1999) Correlation-based feature selection for machine learning

22. Hernando A, Bobadilla J, Ortega F (2016) A non negative matrix factorization for collaborative filtering recommender systems based on a bayesian probabilistic model. Knowl Based Syst 97:188-202

23. Hernando A, Bobadilla J, Ortega F, Gutiérrez A (2013) Trees for explaining recommendations made through collaborative filtering. Inf Sci 239:1-17. https://doi.org/10.1016/j.ins. 2013.03.018

24. Holstein K, Vaughan JW, III HD, Dudík M, Wallach HM (2019) Improving fairness in machine learning systems: What do industry practitioners need? In: Brewster SA, Fitzpatrick G, Cox AL, Kostakos V (eds) Proceedings of the 2019 CHI conference on human factors in computing systems, CHI 2019, Glasgow, Scotland, UK, May 04-09, 2019. ACM, p 600. https://doi.org/10. $1145 / 3290605.3300830$

25. Huang T, Zhang D, Bi L (2020) Neural embedding collaborative filtering for recommender systems. Neural Comput Appl. https:// doi.org/10.1007/s00521-020-04920-9

26. Jiang F, Sui $Y$, Zhou L (2015) A relative decision entropy-based feature selection approach. Pattern Recognit 48(7):2151-2163. https://doi.org/10.1016/j.patcog.2015.01.023

27. Jiang M, Zhang Z, Jiang J, Wang Q, Pei Z (2019) A collaborative filtering recommendation algorithm based on information theory and bi-clustering. Neural Comput Appl 31(12):8279-8287. https://doi.org/10.1007/s00521-018-3959-2

28. Jing Y, Yang Y, Feng Z, Ye J, Yu Y, Song M (2019) Neural style transfer: a review. IEEE Trans Vis Comput Graph pp 1

29. Jolliffe IT (2002) Principal component analysis. Springer, New York. https://doi.org/10.1007/978-0-387-22440-4

30. Krishnaswamy Rangarajan A, Purushothaman R (2020) Disease classification in eggplant using pre-trained VGG16 and MSVM. Sci Rep 10(2322):1-11. https://doi.org/10.1038/s41598-02059108-x

31. Lara-Cabrera R, González-Prieto Á, Ortega F (2020) Deep matrix factorization approach for collaborative filtering recommender systems. Appl Sci 10(14):4926

32. Lee DD, Seung HS (2001) Algorithms for non-negative matrix factorization. In: Leen TK, Dietterich TG, Tresp V (eds) Advances in neural information processing systems 13. MIT Press, pp 556-562

33. Leonhardt J, Anand A, Khosla M (2020) User fairness in recommender systems. Companion Proc Web Conf 2018:101-102. https://doi.org/10.1145/3184558.3186949

34. Li F, Xu G, Cao L (2016) Two-level matrix factorization for recommender systems. Neural Comput Appl 27(8):2267-2278. https://doi.org/10.1007/s00521-015-2060-3

35. Lin M, Chen Q, Yan S (2014) Network in network. CoRR arXiv: abs/1312.4400

36. Lully V, Laublet P, Stankovic M, Radulovic F (2018) Enhancing explanations in recommender systems with knowledge graphs.
Procedia Comput Sci 137:211-222. https://doi.org/10.1016/j. procs.2018.09.020

37. Madadipouya K, Chelliah S (2017) A literature review on recommender systems algorithms, techniques and evaluations. BRAIN Broad Res Artif Intell Neurosci 8(2):109-124

38. Mnih A, Salakhutdinov RR (2008) Probabilistic matrix factorization. In: Advances in neural information processing systems. pp. $1257-1264$

39. $\mathrm{Mu} \mathrm{R}$ (2018) A survey of recommender systems based on deep learning. IEEE Access 6:69009-69022. https://doi.org/10.1109/ ACCESS.2018.2880197

40. Ng AY (2020) Feature selection, L1 vs. L2 regularization, and rotational invariance. In: Proceedings of the twenty-first international conference on machine learning. p 78. https://doi.org/10. $1145 / 1015330.1015435$

41. Nunes I, Jannach D (2017) A systematic review and taxonomy of explanations in decision support and recommender systems. User Model User Adap Interface 27(3):393-444. https://doi.org/10. 1007/s11257-017-9195-0

42. Ortega F, Lara-Cabrera R, González-Prieto Á, Bobadilla J (2020) Providing reliability in recommender systems through Bernoulli matrix factorization. arXiv preprint arXiv:2006.03481

43. Papadimitriou A, Symeonidis P, Manolopoulos Y (2012) A generalized taxonomy of explanations styles for traditional and social recommender systems. Data Min Knowl Discov 24(3):555-583. https://doi.org/10.1007/s10618-011-0215-0

44. Peng H, Long F, Ding C (2005) Feature selection based on mutual information criteria of max-dependency, max-relevance, and min-redundancy. IEEE Trans Pattern Anal Mach Intell 27(8):1226-1238

45. Quijano-Sanchez L, Sauer C, Recio-Garcia JA, Diaz-Agudo B (2017) Make it personal: a social explanation system applied to group recommendations. Expert Syst Appl 76:36-48. https://doi. org/10.1016/j.eswa.2017.01.045

46. Reed R, MarksII RJ (1999) Neural smithing: supervised learning in feedforward artificial neural networks. Mit Press, New York

47. Rezvanian A, Moradabadi B, Ghavipour M, Khomami MMD, Meybodi MR (2019) Social recommender systems. Springer, Berlin, pp 281-313

48. Selvaraju RR, Cogswell M, Das A, Vedantam R, Parik, D, Batra D (2017) Grad-CAM: visual explanations from deep networks via gradient-based localization. In: 2017 IEEE international conference on computer vision (ICCV). pp 618-626 . https://doi.org/10. 1109/ICCV.2017.74

49. Shamshirband S, Rabczuk T, Chau KW (2019) A survey of deep learning techniques: application in wind and solar energy resources. IEEE Access 7:164650-164666

50. Sohail SS, Siddiqui J, Ali R (2017) Classifications of recommender systems: a review. J Eng Sci Technol Rev 10(4):132-153

51. Tsintzou V, Pitoura E, Tsaparas $P$ (2018) Bias disparity in recommendation systems. Preprint: arXiv

52. Valdiviezo-Diaz P, Ortega F, Cobos E, Lara-Cabrera R (2019) A collaborative filtering approach based on Naïve Bayes classifier. IEEE Access 7:108581-108592. https://doi.org/10.1109/ ACCESS.2019.2933048

53. Villegas NM, Sánchez C, Díaz-Cely J, Tamura G (2018) Characterizing context-aware recommender systems: a systematic literature review. Knowl Based Syst 140:173-200

54. Wang Wc, Xu L, Chau Kw, Xu Dm (2020) Yin-yang firefly algorithm based on dimensionally Cauchy mutation. Expert Syst Appl 150:113216

55. Wang X, Qian X (2018) Total variance based feature point selection and applications. Comput Aided Des 101:37-56. https:// doi.org/10.1016/j.cad.2018.04.003

56. Wen L, Li X, Li X, Gao L (2019) A new transfer learning based on vgg-19 network for fault diagnosis. In: 2019 IEEE 23rd 
international conference on computer supported cooperative work in design (CSCWD). pp 205-209

57. Wu C, Chau KW (2013) Prediction of rainfall time series using modular soft computingmethods. Eng Appl Artif Intell 26(3):997-1007

58. Yao S, Huang B (2017) Beyond parity: fairness objectives for collaborative filtering. In: Advances in neural information processing systems. pp 2921-2930

59. Zamani H, Shakery A (2018) A language model-based framework for multi-publisher content-based recommender systems. Inf Retr J 21(5):369-409
60. Zanker M, Ninaus D (2020) Knowledgeable explanations for recommender systems. In: IEEE date of conference: 31 Aug. -3 Sept 2010. https://doi.org/10.1109/WI-IAT.2010.131

61. Zhou B, Khosla A, Lapedriza A, Oliva A, Torralba A (2016) Learning deep features for discriminative localization. In: 2016 IEEE conference on computer vision and pattern recognition (CVPR). pp 2921-2929

Publisher's Note Springer Nature remains neutral with regard to jurisdictional claims in published maps and institutional affiliations. 